\title{
Validation of automated oscillometric versus manual measurement of the ankle-brachial index
}

\begin{abstract}
Tom Richart ${ }^{1,2}$, Tatiana Kuznetsova ${ }^{2}$, Barbara Wizner ${ }^{3}$, Harry A Struijker-Boudier ${ }^{4}$ and Jan A Staessen ${ }^{1,2}$
We validated automated oscillometric measurement of the ankle-to-arm ratio of systolic blood pressure (ankle-brachial blood pressure index, $\mathrm{ABI}$ ) against the manual approach, which requires trained observers and has an intra-observer variability of $\sim 10 \%$. A single observer measured ABI in 105 subjects (age range, 20-80 years; women, 51.9\%). The manual approach involved the auscultatory measurement of systolic pressure at the brachial artery and Doppler measurements of systolic pressure at the anterior and posterior tibial arteries. The automated oscillometric VP-2000 (Omron Healthcare, Kyoto, Japan) was used to measure systolic pressure at the arm and ankle simultaneously. We applied Bland and Altman's methods to study reproducibility. Ankle-brachial blood pressure index averaged ( \pm s.d.) $1.13 \pm 0.07$ units and $1.12 \pm 0.10$ units on automated and manual measurements, respectively. The absolute and percentage differences $(P=0.64)$ between the two $A B I$ measurements were 0.005 units $(95 \%$ confidence interval, -0.015 to 0.024$)$ and 0.60 ( -0.69 to 1.04$)$, respectively. The intra-observer variability of repeat $A B I$ measurements was smaller on automated than on manual measurement $(0.17$ versus $-0.84 \% ; P=0.04)$. Automated $A B I$ measurement by an oscillometric technique requires little observer training, and significantly increases the reproducibility of $A B I$ estimates compared with the manual approach.
\end{abstract}

Hypertension Research (2009) 32, 884-888; doi:10.1038/hr.2009.125; published online 4 September 2009

Keywords: ankle-brachial index; blood pressure measurement; oscillometry; peripheral arterial disease

\section{INTRODUCTION}

The ankle-brachial blood pressure index (ABI) is a simple and noninvasive measure to assess the patency of the arteries of the lower extremities, and predicts cardiovascular morbidity and mortality. ${ }^{1-3}$ $\mathrm{ABI}$ is usually measured by having the patients lie in the supine position with subsequent performance of ankle and brachial blood pressure measurements. Doppler is considered the gold standard for ankle blood pressure measurement in ABI determination. ${ }^{4,5}$ This approach requires trained observers and has an intra-observer variability of $\sim 10 \%{ }^{6-8}$ The use of automated devices might facilitate the assessment of this index and enhance its reproducibility

The VP-2000 (Omron Healthcare) is an automated oscillometric device, designed to measure $\mathrm{ABI}$ from simultaneous 4-cuff blood pressure measurements at the left and right brachial, and left and right tibial arteries. The Food and Drug Administration recently approved the VP2000 device for clinical use in the United States. This device passed validation for both the arm and ankle blood pressure measurements. ${ }^{9}$

To our knowledge, few studies have validated the measurement of ABI by an automated oscillometric technique against the gold standard (Doppler). In this study, a single observer measured ABI in 105 subjects representative of a European population. We compared the reproducibility of the ABI, as measured by the automated and manual techniques.

\section{METHODS}

Study population

In the framework of the Flemish Study on Environment, Genes and Health Outcomes (FLEMENGHO), ${ }^{10}$ we randomly recruited 106 participants in a rural area of Flanders, Belgium. The ethics committee of the University of Leuven approved the study. Eligible subjects had a minimum age of 12 years. All participants gave an informed written consent. We excluded one participant with an ABI of 0.7 from statistical analysis.

\section{Measurements}

A single observer (TR) performed the clinical measurements, and administered a validated questionnaire to obtain information on each participant's medical history, intake of medications, and smoking and drinking habits.

To ensure steady-state conditions, the ABI measurements were obtained in a quiet examination room, after the subjects had rested for $15 \mathrm{~min}$ in the supine position and refrained from smoking, heavy exercise, and drinking alcohol or caffeinated beverages for at least $2 \mathrm{~h}$ before the examination. The observer first obtained two consecutive blood pressure readings to the nearest $2 \mathrm{~mm} \mathrm{Hg}$ at the subjects' right arm, using a standard mercury sphygmomanometer (Riester GmbH, Jungingen, Germany). He applied the Korotkoff technique, as outlined in the 2003 guidelines of the European Society of Hypertension. ${ }^{11}$ Hypertension was a brachial blood pressure of at least $140 \mathrm{~mm} \mathrm{Hg}$ systolic or $90 \mathrm{~mm} \mathrm{Hg}$ diastolic, or the use of antihypertensive drugs. Next, the observer obtained paired Doppler readings of systolic blood

${ }^{1}$ Department of Epidemiology, Maastricht University, Maastricht, The Netherlands; ${ }^{2}$ Studies Coordinating Centre, Division of Hypertension and Cardiac Rehabilitation, Department of Cardiovascular Diseases, University of Leuven, Leuven, Belgium; ${ }^{3}$ Department of Internal Medicine and Gerontology, Jagellonian University Medical College, Kraków, Poland; and ${ }^{4}$ Division of Pharmacology, Maastricht University, Maastricht, The Netherlands

Correspondence: Dr T Richart, Department of Epidemiology, Maastricht University, Peter Debyeplein 1, PO box 616, 6200 MD Maastricht, The Netherlands.

E-mail: tom.richart@epid.unimaas.nl

Received 20 March 2009; revised 26 May 2009; accepted 21 June 2009; published online 4 September 2009 
pressure at the right anterior and posterior tibial arteries, using a hand-held $8 \mathrm{MHz}$ A27116 probe (SonicAid, Oxford Instruments, Surrey, UK). The cuff used for the brachial and posterior tibial blood pressure measurements had a bladder size of $22 \times 32 \mathrm{~cm}$, which was suitable for the circumferences of the upper arm (range, $21.5-32.7 \mathrm{~cm}$ ) and ankle (range, 18.5-26.5 cm) of all participants. Finally, the observer obtained two simultaneous oscillometric blood pressure readings at the right and left brachial, and right and left tibial arteries, using the VP-2000 device. The cuffs for the arm measurements and the pre-shaped cuffs for the measurement at the ankles had bladders with sizes of $20 \times 32$ and $23 \times 33 \mathrm{~cm}$, respectively. We computed the automatically and manually obtained ABIs as the ratios of the ankle-to-brachial blood pressures.

\section{Statistical methods}

For database management and statistical analyses, we used the SAS software, version 9.1.3 (SAS Institute, Cary, NC, USA) with its JMP add-on, version 6.1. We compared the means and proportions by Student's $t$-test for paired and unpaired observations, as appropriate, and the $\chi^{2}$-statistic, respectively. Our statistical methods also included single regression.

We assessed reproducibility by Bland and Altman's approach. For analysis of concordance between the automated and manual techniques, we averaged each pair of measurements by the technique used and considered only the oscillometric measurements obtained at the right arm and ankle. We compared the Doppler measurements of blood pressure at the anterior and posterior tibial arteries, and their average, with the oscillometrically derived ankle pressures. We also assessed the reproducibility of successive measurements at the same site for both the automated and manual approaches.

\section{RESULTS}

\section{Characteristics of the participants}

The 105 participants' age ranged from 20 to 80 years. The brachial blood pressure averaged $129.3 \pm 15.7 \mathrm{~mm} \mathrm{Hg}$ systolic and $77.8 \pm 8.5 \mathrm{~mm} \mathrm{Hg}$ diastolic. The systolic blood pressure at the ankle, computed from the measurements at both the anterior and posterior tibial arteries, averaged $150.7 \pm 18.5$ and $143.7 \pm 20.7 \mathrm{~mm} \mathrm{Hg}$ on oscillometric and Doppler measurements, respectively. Of all participants, $26(24.8 \%)$ were hypertensive, 28 (26.7\%) were current smokers and $20(21.4 \%)$ reported regular alcohol intake. There were no subjects with atrial fibrillation. Table 1 lists the characteristics of the participants, including $\mathrm{ABI}$ on automated and manual measurements by sex. Table 2 provides the blood pressure values by site of measurement. Figure 1 shows the mean values of ABI with 95\% confidence interval (CI) for automated and manual measurements based on the systolic blood pressure at both ankle arteries, and in addition, for the manual measurements based on the systolic blood pressure at either the anterior or posterior tibial artery. There was no difference $(P=0.78)$ between the automated ABI measurements at the right side $(1.11 \pm 0.07)$, which we used in the current analysis, and those at the left side $(1.12 \pm 0.07)$.

\section{Manual versus automated blood pressure measurements}

The auscultatory readings of systolic and diastolic blood pressure at the brachial artery and the Doppler readings of systolic blood pressure at the anterior and posterior tibial arteries were significantly $(P \leqslant 0.0001)$ lower than the corresponding oscillometric readings at the same site (Table 2).

Estimates of ABI based on automated and manual measurements tended to be different when the manual measurement of systolic blood pressure at the ankle rested on a single artery, but not when the Doppler measurements of systolic blood pressure at the anterior and posterior tibial arteries were averaged to compute the manually measured ABI. (Figure 2 and Table 2) The automated-manual differences averaged 0.029 units (95\% CI, 0.007 to 0.051 units;
Table 1 Characteristics of participants by sex

\begin{tabular}{lccc}
\hline & Women $(\mathrm{n}=55)$ & Men $(\mathrm{n}=50)$ & P-values \\
\hline Age (y) & $57.7 \pm 12.6$ & $55.1 \pm 14.8$ & 0.33 \\
Brachial systolic pressure $(\mathrm{mm} \mathrm{Hg})$ & $125.7 \pm 16.4$ & $133.1 \pm 14.1$ & 0.017 \\
Brachial diastolic pressure $(\mathrm{mm} \mathrm{Hg})$ & $74.8 \pm 7.4$ & $81.2 \pm 8.4$ & $<0.0001$ \\
Pulse rate $\left(\mathrm{min}^{-1}\right)$ & $64.9 \pm 8.4$ & $62.1 \pm 8.6$ & 0.09 \\
Body height $(\mathrm{cm})$ & $161.3 \pm 7.1$ & $175.8 \pm 7$ & $<0.0001$ \\
Body weight $(\mathrm{kg})$ & $67.9 \pm 12.5$ & $81.5 \pm 8.9$ & $<0.0001$ \\
Body mass index $\left(\mathrm{kg} \mathrm{m}^{-2}\right)$ & $26 \pm 4.1$ & $26.4 \pm 3$ & 0.55 \\
Waist-to-hip ratio & $0.86 \pm 0.06$ & $0.94 \pm 0.07$ & $<0.0001$ \\
Hypertensive & $12(22 \%)$ & $14(28 \%)$ & 0.63 \\
Smokers & $13(26 \%)$ & $15(28 \%)$ & 0.73 \\
Drinkers & $4(8 \%)$ & $16(30 \%)$ & $<0.0001$
\end{tabular}

Ankle-to-brachial index

Manual technique

Both arteries

Dorsalis pedis

$1.11 \pm 0.10 \quad 1.13 \pm 0.09 \quad 0.32$

Tibialis posterior

$1.08 \pm 0.11 \quad 1.1 \pm 0.10$

0.21

$1.14 \pm 0.11 \quad 1.15 \pm 0.10$

0.64

Automated technique

$\begin{array}{llll}\text { Left } & 1.12 \pm 0.07 & 1.13 \pm 0.08 & 0.75 \\ \text { Right } & 1.11 \pm 0.07 & 1.11 \pm 0.07 & 0.71\end{array}$

Values are mean \pm s.d. or number of subjects (\%). Hypertension was a brachial blood pressure of at least $140 \mathrm{~mm} \mathrm{Hg}$ systolic or $90 \mathrm{~mm} \mathrm{Hg}$ diastolic, or the use of antihypertensive drugs. $P$-values are for the sex difference.

$P=0.01),-0.019$ units $(\mathrm{CI},-0.040$ to 0.080 units; $P=0.08)$ and 0.005 units $(\mathrm{CI},-0.015$ to 0.024 units; $P=0.64$ ) if the ankle systolic blood pressure rested on the anterior tibial artery, the posterior tibial artery or on both arteries, respectively. We did not notice any influence of the cuff size either on the concordance between the oscillometric and Doppler measurements at the ankles $(P=0.71)$ or on the oscillometric blood pressure measured at the arms and the auscultatory measurements at the arm $(P=0.79)$.

As substantiated by the plots (Figure 2), the differences between the oscillometric and manual approaches vary in function of the ABI. At lower values, the $\mathrm{ABI}$ was higher when determined oscillometrically, whereas at ABI values above 1.1, the Doppler-derived ABI was higher than the oscillometrically derived index.

In single regression analyses, we could not identify any significant association $(0.10<P<0.62)$ between the automated minus manual measurements (both ankle arteries) and possible explanatory variables, including sex, age, body mass index, pulse rate, and systolic or diastolic brachial blood pressure on auscultatory or oscillometric measurement.

\section{Intra-observer reproducibility of repeat measurements}

Table 3 shows the repeatability of two consecutive measurements of systolic blood pressure at the same site and that of two consecutive ABI measurements. Although the first and repeated measurements were on average similar for the automated technique $(P \geqslant 0.33)$, the manual approach resulted in slightly higher estimates of $A B I$ on repeat compared with the first measurement. However, the difference only reached statistical significance for the ABI involving Doppler measurements at both ankle arteries ( 0.01 units; $95 \%$ CI, 0.0002 to 0.019 units; $P=0.04$ ). The slightly higher systolic blood pressure on repeat than on the first measurement explained the latter observation $(0.81 ; 0.05$ to $1.59 \mathrm{~mm} \mathrm{Hg} ; P=0.04)$. The intra-observer variability of repeat $\mathrm{ABI}$ measurements was smaller on automated than manual measurement (0.17 versus $-0.84 \% ; P=0.04)$. 
Table 2 Bland and Altman analysis of manual versus automated blood pressure measurements

\begin{tabular}{|c|c|c|c|c|c|c|c|}
\hline \multirow[b]{3}{*}{ Measurement } & \multicolumn{2}{|c|}{ Mean value ( \pm s.d.) } & \multicolumn{2}{|c|}{ Absolute difference } & \multicolumn{2}{|c|}{ Relative difference ${ }^{a}$} & \multirow[b]{3}{*}{ Prob $>|t|^{\mathrm{b}}$} \\
\hline & \multirow[b]{2}{*}{ Manualc $^{\mathrm{c}}$} & \multirow[b]{2}{*}{ Automated $^{\mathrm{d}}$} & \multicolumn{2}{|c|}{ Automated minus manual } & \multicolumn{2}{|c|}{ Automated minus manual } & \\
\hline & & & Mean ( \pm s.d.) & $95 \% \mathrm{Cl}$ & Mean ( \pm s.d.) & $95 \% \mathrm{Cl}$ & \\
\hline \multicolumn{8}{|c|}{ Arm blood pressure ( $\mathrm{mm} \mathrm{Hg}$ ) } \\
\hline Systolic pressure & $129.3 \pm 15.7$ & $133.1 \pm 14.8$ & $3.84 \pm 10.07$ & 5.79 to 1.89 & $3.04 \pm 7.43$ & -1.60 to 4.47 & 0.0001 \\
\hline Diastolic pressure & $77.8 \pm 8.5$ & $78.4 \pm 9.7$ & $0.59 \pm 6.60$ & 1.87 to -0.68 & $0.56 \pm 8.85$ & -1.15 to 2.27 & 0.0001 \\
\hline \multicolumn{8}{|c|}{ Ankle blood pressure $(\mathrm{mm} \mathrm{Hg})^{e}$} \\
\hline Dorsalis pedis & $141.2 \pm 21.4$ & $150.7 \pm 18.5$ & $11.18 \pm 14.39$ & 13.98 to 8.38 & $7.99 \pm 10.42$ & 5.96 to 10.02 & $<0.0001$ \\
\hline Tibialis Posterior & $147.4 \pm 20.2$ & $150.7 \pm 18.5$ & $5.15 \pm 12.97$ & 7.66 to 2.64 & $3.59 \pm 8.86$ & 1.88 to 5.31 & $<0.0001$ \\
\hline Both arteries & $143.7 \pm 20.7$ & $150.7 \pm 18.5$ & $8.17 \pm 12.40$ & 10.7 to 5.76 & $5.69 \pm 8.64$ & 4.01 to 7.37 & $<0.0001$ \\
\hline \multicolumn{8}{|l|}{$A B I$} \\
\hline Dorsalis pedis & $1.10 \pm 0.11$ & $1.13 \pm 0.07$ & $0.029 \pm 0.01$ & 0.051 to 0.007 & $2.90 \pm 10.49$ & 0.86 to 4.94 & $0.011^{c}$ \\
\hline Tibialis Posterior & $1.14 \pm 0.11$ & $1.13 \pm 0.07$ & $-0.019 \pm 0.009$ & -0.04 to 0.08 & $1.41 \pm 9.50$ & -3.25 to 0.43 & 0.08 \\
\hline Both arteries & $1.12 \pm 0.10$ & $1.13 \pm 0.07$ & $0.005 \pm 0.001$ & 0.024 to -0.015 & $0.60 \pm 9.00$ & -1.15 to 2.35 & 0.64 \\
\hline
\end{tabular}

Abbreviations: $\mathrm{ABI}$, ankle-brachial index; $\mathrm{Cl}$, confidence interval; Prob, probability.

aRelative difference was computed from paired readings as $(x 1-x 2) /[(x 1+x 2) / 2] \times 100$; the differences between automated and manual measurements were calculated using Bland and Altman's approach.

bProb $>\mid$ t| for Bland-Altman's approach (matched pairs), comparing manual and automated measurements.

cManual blood pressure is the mean of two successive auscultatory measurements (right arm) or of two successive Doppler measurements (right ankle).

${ }^{d}$ Automated blood pressure is the mean of two successive oscillometric measurements (right arm and ankle). The automated approach simultaneously assesses the blood pressure at the anterior and posterior ankle arteries.

eDoppler measurements of ankle systolic blood pressure at the right ankle.

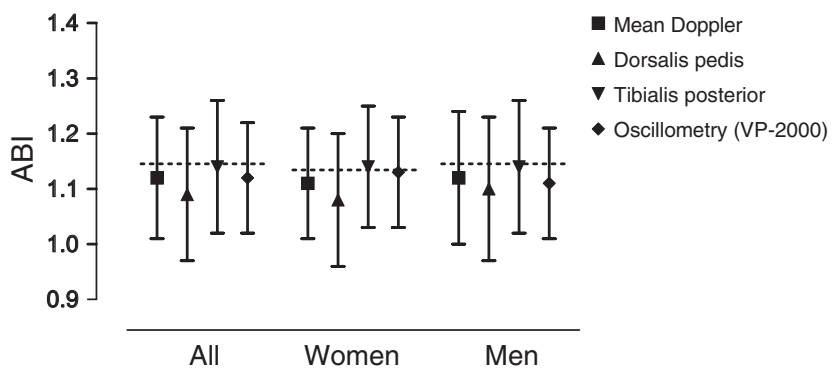

Figure 1 Concordance of ankle-brachial blood pressure index (ABI) measured by auscultatory and oscillometric methods. Plotted values are means $(95 \% \mathrm{Cl})$ of $\mathrm{ABI}$ in 55 women, 50 men and in all 105 participants.

- Auscultatory measurements at the anterior dorsalis pedis and anterior tibialis posterior; $\boldsymbol{\Delta}$, auscultatory measurements at the anterior dorsalis pedis; $\boldsymbol{\nabla}$, auscultatory measurements at the anterior tibialis posterior; $\bullet$, oscillometric measurements (VP-2000).

\section{DISCUSSION}

In this study, we compared the Doppler measurements of ABI with an observer-free automated oscillometric method in a general Flemish population sample. Our key finding was that an automated ABI measurement by means of the VP-2000 device in an epidemiological setting is feasible. Successive measurements using the automated device had better repeatability than did the manual techniques at all measurement sites. The significant difference between repeated measurements of the manual ABI taking both arteries into account is probably attributable to the higher number of measurements needed for its calculation (two times, three measurements as opposed to two times, two for the single-artery indices) (Table 3).

The automatically obtained ABI values were concordant with those obtained using the gold standard, that is measurement at the ankle over the tibialis posterior or both arteries, using the highest value. On the other hand, the mean arm and ankle blood pressure values were significantly higher in automated measurements compared with manual auscultatory and Doppler measurements, possibly attributable to the intrinsic differences in the oscillometric and manual blood pressure measurement techniques.

Cortez-Cooper et al. ${ }^{9}$ compared, in 52 normotensive and hypertensive women and men, the oscillometric and manual measurements of systolic blood pressure at the posterior tibial artery, using the VP2000 device and a hand-held Doppler flow meter. Ankle systolic blood pressures measured using the oscillometric machine $(142 \pm 23 \mathrm{~mm} \mathrm{Hg})$ were highly correlated with those obtained using the Doppler probe $(145 \pm 21 \mathrm{~mm} \mathrm{Hg})$, although the regression line deviated slightly from the line of identity. The correlation coefficient was 0.95 with an average difference in the means of $2.2 \pm 6.8 \mathrm{~mm} \mathrm{Hg} .{ }^{9}$ In keeping with our present findings, the Doppler measurements were higher than the oscillometric readings. Cortez-Cooper also observed an inverse association between the differences of the Doppler minus the oscillometric systolic values and the height of systolic pressure. In our study, the corresponding correlation coefficient was 0.14 $(P<0.0001)$.

Several investigators used Dinamap devices to measure $A B I$ in normal volunteers or in patients with various degrees of peripheral arterial disease (PAD). ${ }^{12}$ On balance, these studies showed only weak agreement between the Dinamap and Doppler techniques in normal volunteers and in patients without arterial disease or with only minor obstructive arterial lesions. However, in patients with significant stenosis, the sensitivity of the Dinamap device was insufficient to reliably measure the ankle blood pressure and to evaluate ABI. This is not surprising because the Dinamap machines were either not validated or failed to pass validation according to the current guidelines on blood pressure measurement. Rose et al. ${ }^{13}$ even found that two types of Dinamap machines systematically skipped 14 values of 
a
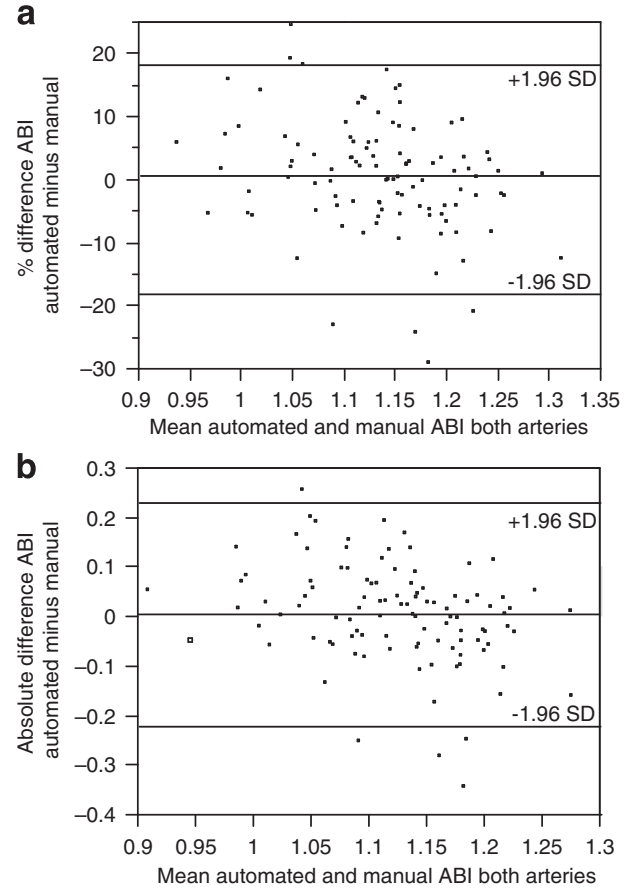

C

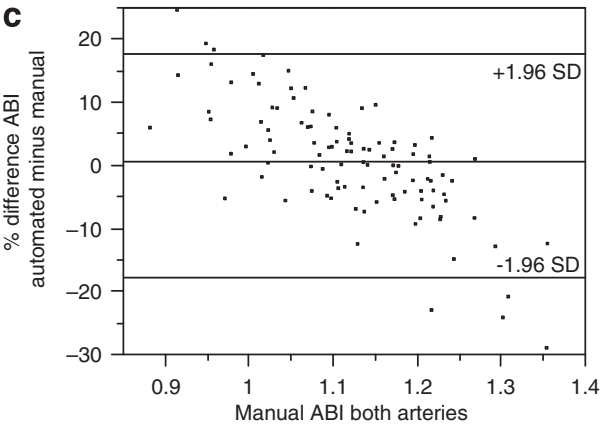

d

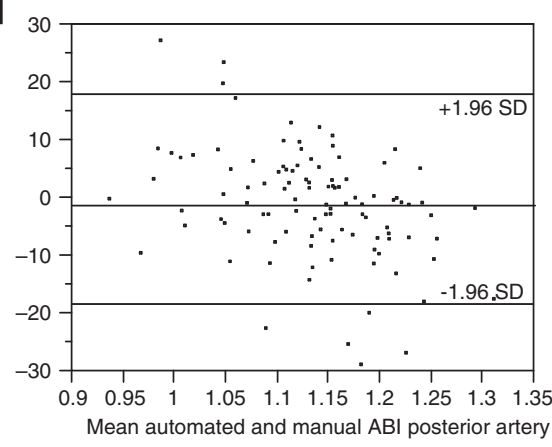

e 0

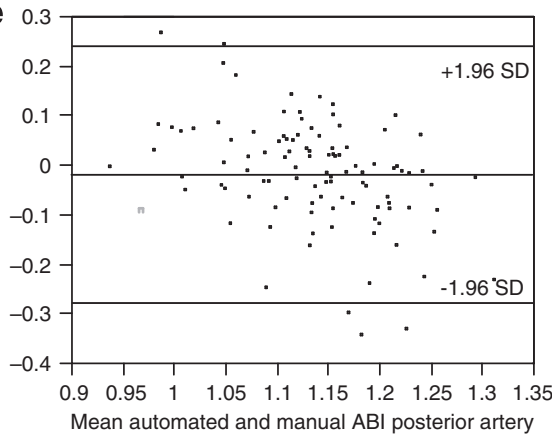

f

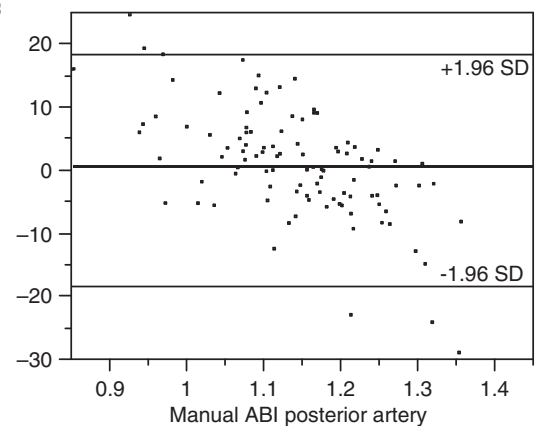

Figure 2 Bland and Altman plots for the differences between automated and manual ankle-brachial blood pressure index (ABI) measurements. (a-c) ABI derived at the anterior and posterior tibial arteries. (d-f) $A B I$ derived at the posterior tibial artery. (a and $\mathbf{d})$ Percent difference in ABI between automated minus manual technique vs. mean $A B I$ based on both techniques. ( $b$ and $\mathbf{e}$ ) Absolute difference in $A B I$ between automated minus manual technique vs. mean $\mathrm{ABI}$ based on both techniques. (c and $\mathbf{f}$ ) Percentage difference in $\mathrm{ABI}$ between automated minus manual technique vs. $\mathrm{ABI}$ based on the manual technique.

Table 3 Bland and Altman analysis of consecutive measurements by the same observer

\begin{tabular}{|c|c|c|c|c|c|}
\hline \multirow[b]{2}{*}{ Measurement } & \multicolumn{2}{|c|}{ Absolute difference second-first measurement } & \multicolumn{2}{|c|}{ Relative difference second-first measurement } & \multirow[b]{2}{*}{ Prob $>|t|^{\mathrm{a}}$} \\
\hline & Mean ( \pm s.d.) & $95 \% \mathrm{Cl}$ & Mean ( \pm s.d.) & $95 \% \mathrm{Cl}$ & \\
\hline Systolic brachial pressure & $0.81 \pm 3.98$ & 1.59 to 0.05 & $0.62 \pm 3.98$ & -6.74 to 1.23 & 0.037 \\
\hline Diastolic brachial pressure & $-0.18 \pm 3.25$ & 0.43 to -0.82 & $-0.38 \pm 2.58$ & -1.74 to 0.91 & 0.55 \\
\hline Systolic tibialis posterior & $-0.34 \pm 5.05$ & 1.29 to -0.65 & $-0.22 \pm 3.47$ & -0.89 to -0.46 & 0.48 \\
\hline ABI dorsalis pedis & $0.01 \pm 0.05$ & 0.10 to 0 & $-0.80 \pm 4.71$ & -1.72 to 0.11 & 0.095 \\
\hline ABI both arteries & $0.01 \pm 0.05$ & 0.019 to 0.0002 & $-0.84 \pm 4.23$ & -1.66 to -0.02 & 0.04 \\
\hline \multicolumn{6}{|l|}{ Automated } \\
\hline Systolic brachial pressure & $0.029 \pm 6.13$ & 1.51 to -0.86 & $-0.19 \pm 4.57$ & -1.08 to 0.69 & 0.59 \\
\hline Systolic ankle pressure & $-0.31 \pm 8.59$ & 1.34 to -1.97 & $0.20 \pm 5.80$ & -0.93 to 1.32 & 0.71 \\
\hline$A B I$ right side & $0.004 \pm 0.05$ & 0.013 to -0.005 & $0.17 \pm 4.46$ & -0.69 to 1.04 & 0.33 \\
\hline
\end{tabular}

Abbreviations: $\mathrm{ABI}$, ankle-brachial index; $\mathrm{Cl}$, confidence interval; Prob, probability.

aProb $>|t|$ for Bland-Altman's approach (matched pairs), comparing first and second measurements at the same site. 
systolic blood pressure, of which some (140 and $160 \mathrm{~mm} \mathrm{Hg})$ are critical in the diagnosis and treatment of hypertension. We did not detect such phenomenon in the systolic blood pressure readings of the VP-2000 device.

This study must be interpreted within the context of its potential limitations. First, we performed the validation study in a random population sample with low incidence of peripheral arterial disease (PAD). Future studies aimed at the validation of this device in patients affected by PAD are necessary. Second, we determined the manual $\mathrm{ABI}$ indexes only at the right side. For this reason, we discarded in our primary analyses only the oscillometric measurements obtained at the left side. However, our findings remained consistent if we considered the oscillometric measurements obtained at the four limbs (data not shown). Third, the Doppler ABI was the systolic blood pressure ratio of Doppler readings at the posterior tibial artery and/or dorsalis pedis artery, and auscultatory measurements at the brachial artery. By doing so, we mixed two manual measurement techniques. However, the main purpose of using the Doppler measurement at the two arteries at the ankle was to minimize the failure rate of obtaining the ankle blood pressure as well as to better compare the two techniques.

Several studies in the Western and Asian populations have proved that $\mathrm{ABI}$ is a sensitive predictor of cardiovascular outcome. Experts therefore recommended the routine measurement of $\mathrm{ABI}$ in clinical practice for risk stratification and the timely prevention of cardiovascular complications. Automated oscillometric ABI measurement provides a simple method to reach this goal. The technique has the advantage of requiring little observer training and is also free of observer bias. Its validity has been assessed for office use. ${ }^{14}$ Our study showed that there were small differences in the ABI values from oscillometric and manual measurements, which are dependent on the ABI level and must be accounted for in the interpretation of study results.

\section{CONFLICT OF INTEREST}

The authors declare no conflict of interest.

\section{ACKNOWLEDGEMENTS}

The European Union (grants IC15-CT98-0329-EPOGH, LSHM-CT-2006037093 InGenious HyperCare, and HEALTH-F4-2007-201550 HyperGenes) the Fonds voor Wetenschappelijk Onderzoek Vlaanderen, Ministry of the Flemish Community, Brussels, Belgium (grants G.0424.05, G.0575.06 and G.0256.05) and the Katholieke Universiteit Leuven, Belgium (grants OT/99/28, OT/00/25 and OT/05/49) gave support to the Studies Coordinating Centre. The bilateral scientific and technological collaboration between Poland and Flanders, Ministry of the Flemish Community, Brussels (grant BIL/05/22) supported the fellowship of Barbara Wizner in Leuven. OMRON Healthcare (Kyoto, Japan) provided the VP-2000 device for use in the Flemish Study on Environment, Genes and Health Outcomes. The authors gratefully acknowledge the expert assistance of Sandra Covens, Linda Custers, Marie-Jeanne Jehoul and Hanne Truyens (Leuven, Belgium).

1 McDermott MM, Ferrucci L, Simonsick EM, Balfour J, Fried L, Ling S, Gibson D, Guralnik JM. The ankle brachial index and change in lower extremity functioning over time: the Women's Health and Aging Study. J Am Geriatr Soc 2002; 50: 238-246.

2 Spacil J, Spacabilova J. The ankle-brachial blood pressure index as a risk indicator of generalized atherosclerosis. Semin Vasc Med 2002; 2: 441-445.

3 Murabito JM, Guo CY, Fox CS, D'Agostino RB. Heritability of the ankle-brachial index: the Framingham Offspring study. Am J Epidemiol 2006; 164: 963-968.

4 Hirsch AT, Haskal ZJ, Hertzer NR, Bakal CW, Creager MA, Halperin JL, Hiratzka LF, Murphy WR, Olin JW, Puschett JB, Rosenfield KA, Sacks D, Stanley JC, Taylor LM, White CJ, White J, White RA, Antman EM, Smith SC, Adams CD, Anderson JL, Faxon D, Fuster V, Gibbons RJ, Hunt SA, Jacobs AK, Nishimura R, Ornato JP, Page RL, Riegel B. ACC/AHA Guidelines for the Management of Patients with Peripheral Arterial Disease (lower extremity, renal, mesenteric, and abdominal aortic): a collaborative report from the American Associations for Vascular Surgery/Society for Vascular Surgery, Society for Cardiovascular Angiography and Interventions, Society for Vascular Medicine and Biology, Society of Interventional Radiology, and the ACC/AHA Task Force on Practice Guidelines (writing committee to develop guidelines for the management of patients with peripheral arterial disease)-summary of recommendations. J Vasc Interv Radiol 2006; 17: 1383-1397.

5 Management of peripheral arterial disease (PAD). TransAtlantic Inter-Society Consensus (TASC). Eur J Vasc Endovasc Surg 2000; 19(Suppl A): Si-250.

6 Ray SA, Srodon PD, Taylor RS, Dormandy JA. Reliability of ankle: brachial pressure index measurement by junior doctors. Br J Surg 1994; 81: 188-190.

7 Weatherley BD, Chambless LE, Heiss G, Catellier DJ, Ellison CR. The reliability of the ankle-brachial index in the Atherosclerosis Risk in Communities (ARIC) study and the NHLBI Family Heart Study (FHS). BMC Cardiovasc Disord 2006; 6: 7.

8 Holland-Letz T, Endres HG, Biedermann S, Mahn M, Kunert J, Groh S, Pittrow D, Von Bilderling P, Sternitzky R, Diehm C. Reproducibility and reliability of the ankle-brachial index as assessed by vascular experts, family physicians and nurses. Vasc Med 2007; 12: $105-112$.

9 Cortez-Cooper MY, Supak JA, Tanaka H. A new device for automatic measurements of arterial stiffness and ankle-brachial index. Am J Cardiol 2003; 91: 1519-1522, A9.

10 Tikhonoff V, Kuznetsova T, Stolarz K, Bianchi G, Casiglia E, Kawecka-Jaszcz K, Nikitin $\mathrm{Y}$, Tizzone L, Wang JG, Staessen JA. Blood pressure phenotypes in relation to the betaadducin C1797T polymorphism in the European Project on Genes in Hypertension (EPOGH). Blood Press Monit 2003; 8: 151-154.

11 Cifkova R, Erdine S, Fagard R, Farsang C, Heagerty AM, Kiowski W, Kjeldsen S, Luscher T, Mallion JM, Mancia G, Poulter N, Rahn KH, Rodicio JL, Ruilope LM, van Zwieten P, Waeber B, Williams B, Zanchetti A. Practice guidelines for primary care physicians: 2003 ESH/ESC hypertension guidelines. J Hypertens 2003; 21: 1779-1786.

12 Ramanathan A, Conaghan PJ, Jenkinson AD, Bishop CR. Comparison of ankle-brachial pressure index measurements using an automated oscillometric device with the standard Doppler ultrasound technique. ANZ J Surg 2003; 73: 105-108.

13 Rose KM, Arnett DK, Ellison RC, Heiss G. Skip patterns in DINAMAP-measured blood pressure in 3 epidemiological studies. Hypertension 2000; 35: 1032-1036.

14 Beckman JA, Higgins CO, Gerhard-Herman M. Automated oscillometric determination of the ankle-brachial index provides accuracy necessary for office practice. Hypertension 2006; 47: 35-38. 